\title{
Corrigendum to "Quantum-to-the-Home: Achieving Gbits/s Secure Key Rates via Commercial Off-the-Shelf Telecommunication Equipment"
}

\author{
Rameez Asif $\mathbb{D}^{1,2}$ and William J. Buchanan ${ }^{1,2}$ \\ ${ }^{1}$ Centre for Distributed Computing, Networks, and Security, School of Computing, Edinburgh Napier University, \\ Edinburgh EH10 5DT, UK \\ ${ }^{2}$ The Cyber Academy, Edinburgh Napier University, Edinburgh EH10 5DT, UK \\ Correspondence should be addressed to Rameez Asif; r.asif@napier.ac.uk
}

Received 19 May 2019; Accepted 12 June 2019; Published 30 October 2019

Copyright (c) 2019 Rameez Asif and William J. Buchanan. This is an open access article distributed under the Creative Commons Attribution License, which permits unrestricted use, distribution, and reproduction in any medium, provided the original work is properly cited.

The article titled "Quantum-to-the-Home: Achieving Gbits/s Secure Key Rates via Commercial Off-the-Shelf Telecommunication Equipment" [1] was found to contain materials from the following published article that was cited but not properly attributed [2]: "Z. Qu, I. B. Djordjevic, M. A. Neifeld, "RF-subcarrier-assisted Four-state Continuousvariable QKD Based on Coherent Detection," Optics Letters, vol. 41, no. 23, pp. 5507-5510, 2016" [3]. The mathematical models and their description in appendices A and B in [1] were taken from [3] with some rewording of the text, and Figure 2 in [1] is similar to part of Figure 1 in [3].

\section{References}

[1] R. Asif and W. J. Buchanan, "Quantum-to-the-home: achieving gbits/s secure key rates via commercial off-the-shelf telecommunication equipment," Security and Communication Networks, vol. 2017, Article ID 7616847, 10 pages, 2017.

[2] Q. B. Zhen and I. B. Djordjevic, "Comment on "quantum-tothe-home: achieving gbits/s secure key rates via commercial offthe-shelf telecommunication equipment"', Security and Communication Networks, vol. 2019, Article ID 1484593, 1 page, 2019.

[3] Z. Qu, I. B. Djordjevic, and M. A. Neifeld, "RF-subcarrierassisted four-state continuous-variable QKD based on coherent detection," Optics Letters, vol. 41, no. 23, pp. 5507-5510, 2016. 


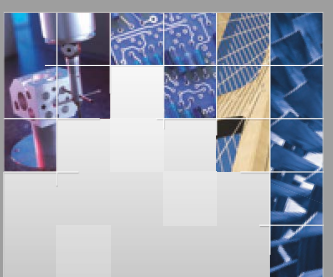

\section{Enfincering}
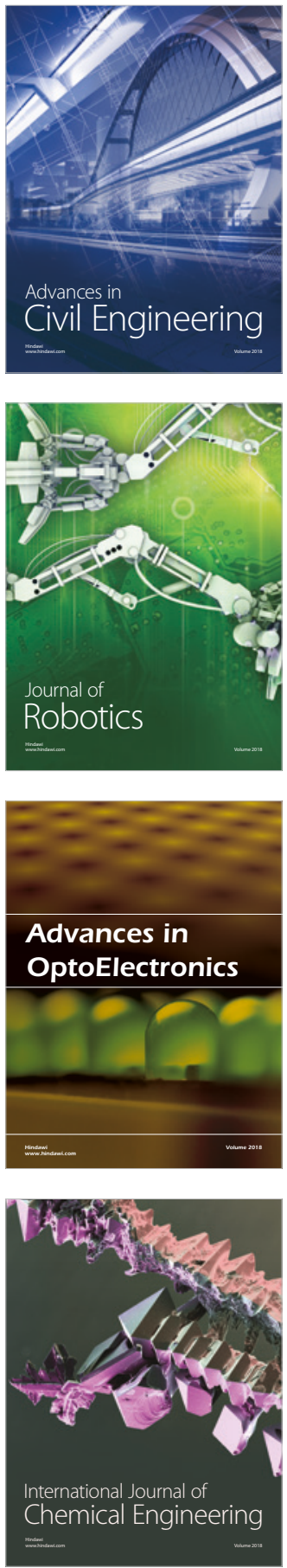

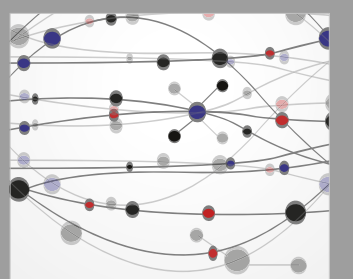

\section{Rotating \\ Machinery}

The Scientific World Journal

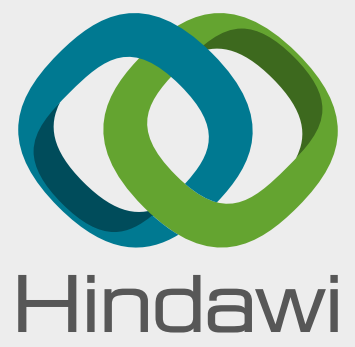

Submit your manuscripts at

www.hindawi.com
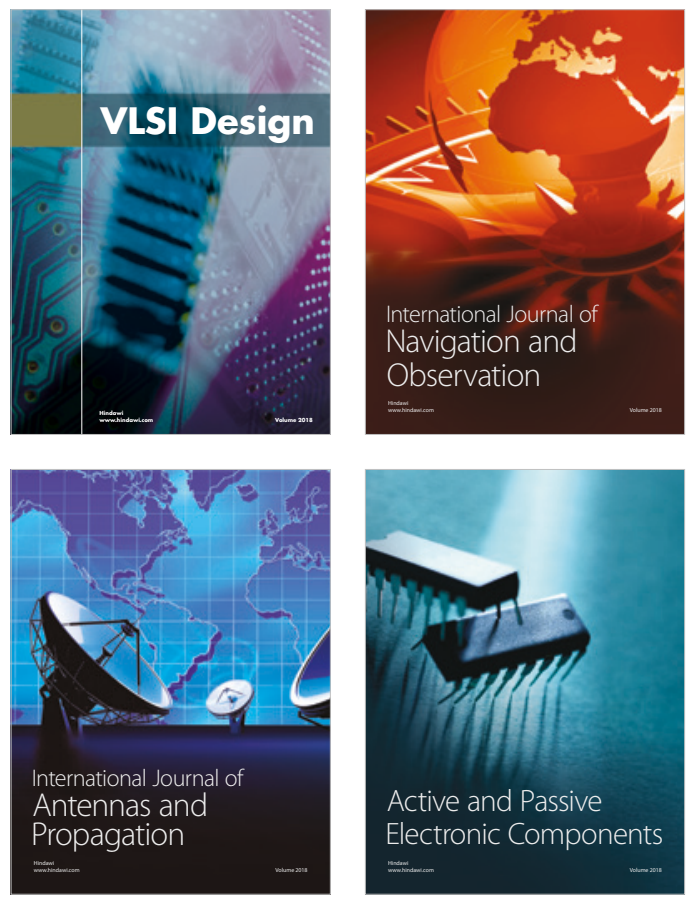
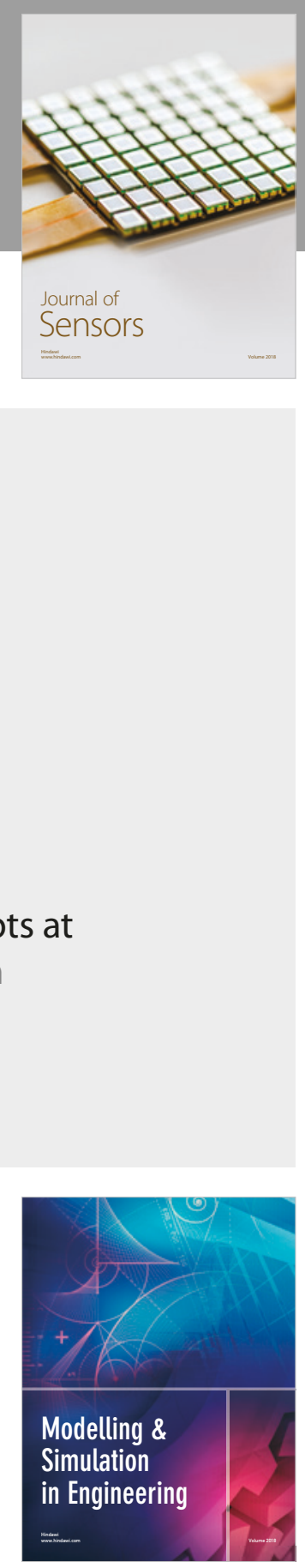

\section{Advances \\ Multimedia}
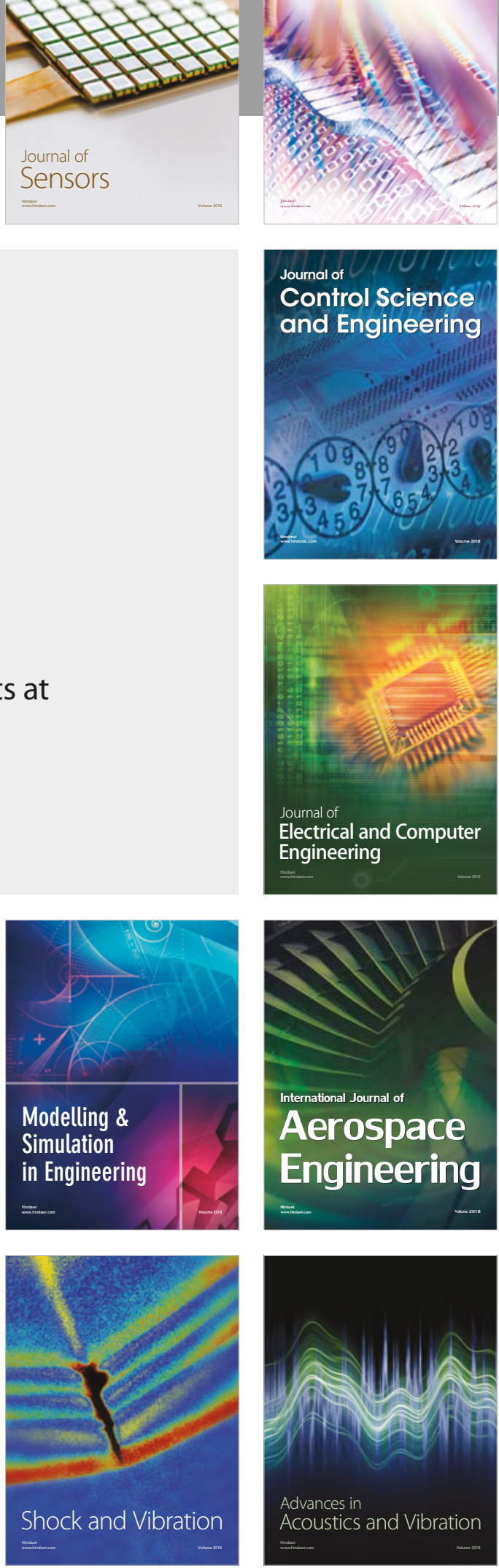